\title{
IMPACTO DO DESMATAMENTO DE UMA ÁREA DE MANGUE NO ALBEDO SUPERFICIAL
}

\section{CARLOS ALEXANDRE SANTOS QUERINO ${ }^{1}$, MARCOS ANTONIO LIMA MOURA², JULIANE KAYSE ALBUQUERQUE DA SILVA QUERINO ${ }^{1}$}

\author{
${ }^{1}$ Universidade Federal do Amazonas, Instituto de Educação, Agricultura e Ambiente (UFAM/IEAA), \\ Humaitá, AM, Brasil \\ ${ }^{2}$ Universidade Federal de Alagoas, Instituto de Ciências Atmosférica (UFAL/ICA), Maceió, AL, Brasil
}

casquerino@ufam.edu.br,malm@ccen.ufal.br, julianekayse@hotmail.com

Recebido Julho de 2012 - Aceito Março de 2013

\begin{abstract}
RESUMO
Manguezais são ecossistemas peculiares encontrados nas regiões tropicais. A degradação dos manguezais altera o balanço superficial de radiação, e por consequência o albedo. Para avaliar e comparar o albedo, nesse ambiente foram instaladas duas plataformas de coletas de dados micrometeorológicos no município de Marechal Deodoro, Alagoas, Brasil, no período de outubro de 2004 a outubro de 2005 . No mangue nativo ( $9^{\circ} 42^{\prime} 18^{\prime \prime} \mathrm{S} ; 35^{\circ} 48^{\prime} 32^{\prime \prime} \mathrm{W}$ ) foram instalados dois piranômetros acima da copa das árvores, e em outubro de 2005, um terceiro dentro do mangue. $\mathrm{Na}$ área degradada $\left(9^{\circ} 36^{\prime} 38^{\prime} \mathrm{S} ; 35^{\circ} 46^{\prime} 03^{\prime}\right.$ ' W), os sensores foram posicionados a uma altura de dois metros em relação ao solo. Observou-se que o albedo sobre a floresta de mangue, em geral, é maior em média, 5 pontos percentuais superior em relação à outras florestas tropicais, como por exemplo, a Amazônia. Internamente notou-se que o mesmo não ultrapassou os $13 \%$ e seu valor máximo ocorre no horário de menor albedo da copa $\approx 20 \%$, evidenciando a influência da maré. Já na área degradada, o albedo médio foi de $35 \%$, o que implica em uma elevação aproximada de $49 \%$ quando substituída a cobertura de floresta natural.
\end{abstract}

Palavras chave: Mangue, Albedo, Radiação Solar.

\begin{abstract}
IMPACT OF MANGROVE DEFORESTATION ON THE SURFACE ALBEDO
Mangroves are peculiar ecosystem and it is found at tropical regions. The degradation of mangroves changes the surface radiation balance, consequently, the albedo. To evaluate and compare albedo data two micrometeorological steal towers was installed in the Marechal Deodoro city, Alagoas, Brazil from October 2004 to October 2005. On the native mangrove tower ( $9^{\circ} 42^{\prime} 18^{\prime \prime} \mathrm{S} ; 35^{\circ} 48^{\prime} 32^{\prime \prime} \mathrm{W}$ ) two pyranometers were fixed above the canopy to collected incident and reflected solar radiation, and in October 2005, a third pyranometer was installed inside of the mangrove area. On the deforested area $\left(9^{\circ} 36^{\prime} 38^{\prime \prime} \mathrm{S} ; 35^{\circ} 46^{\prime} 03^{\prime \prime} \mathrm{W}\right)$ radiation sensors were installed at a higher $2 \mathrm{~m}$ above the surface. The results had shown that in average the albedo above mangrove forest is $5 \%$ higher than over others kind of tropical forest, as an example, Amazonian rain forest. The maximum registered internal albedo was $13 \%$ and it was reached when canopy albedo was at lowest $\approx 20 \%$, evidencing the tilde influence. Moreover, at the deforested area albedo was, in average 35\%, which implies an increase of $49 \%$ when the natural area is deforested.
\end{abstract}

Keywords: Mangroves, Albedo and Solar Radiation. 


\section{INTRODUÇÃO}

As florestas de mangue são detentoras de uma rica estrutura peculiar que abriga uma grande variedade de plantas, animais e microorganismos (Ribeiro et al., 2002; Moura e Querino, 2010; Querino et al., 2011; Moura e Querino, 2012; Sathia e Sekar, 2012). Os manguezais também funcionam como berçário e ponto de alimentação para várias espécies de peixes (Mendonza-Carranza et al., 2010; Sweetman et al., 2010; Osório et al., 2011) tendo, dessa forma, papel fundamental na sustentabilidade de comunidades pesqueiras. Os mangues são encontrados em regiões costeiras tropicais e subtropicais, que cobrem uma área total de aproximadamente 17 milhões de hectares (Hogarth, 2007; Mendonza-Carranza et al., 2010; Sweetman et al., 2010; Sathia e Sekar, 2012). Por possuir grande parte de seu território em região tropical, o Brasil possui uma das maiores áreas de mangue do mundo, que se estende desde o extremo norte até o sul do país. No entanto, a maior concentração de manguezais encontra-se nos litorais do Amapá e Pará, com algumas ocorrências importantes nos estuários do Nordeste (Ribeiro et al., 2002).

Atualmente, uma das grandes questões ambientais diz respeito ao desmatamento das áreas de florestas nativas. Sathia e Sekar (2012) relatam que a degradação dos mangues aumenta a mortandade de peixes, reduz a purificação da água e eleva a salinidade dos solos costeiros. Essas alterações em florestas de mangues causam instabilidades da biodiversidade, do meio físico e por consequência do microclima (Galvani e de Lima, 2011). Por sua vez, o clima e o microclima têm como principal variável controladora o albedo (Querino et al., 2006; Moura et al., 1999).

Albedo é a razão entre o fluxo de radiação refletido por uma dada superfície em todas as direções e o fluxo incidente sobre ela (Moura, 2000; Querino et al., 2006; Kushari e Konitpong, 2011). Ele controla diretamente a partição da energia que chega à superfície, pois representa a habilidade da mesma de refletir energia em todo espectro solar (Kushari e Konitpong, 2011). Dessa maneira, o albedo é responsável por praticamente todos os processos de ordem física, fisiológica e bioquímica dos ecossistemas. O albedo é, portanto, uma medida adimensional que varia segundo as características superficiais (cobertura do solo e sua umidade), das espécies cultivadas, que implicará em variações no arranjo foliar, na razão entre a radiação solar direta e a difusa e em função do ângulo zenital, que varia de acordo com a época do ano e hora do dia (Stanhill et al., 1966; Correia et al., 2002; Querino et al., 2006; Kushari e Konitpong, 2011).

Pinker et al. (1980) observaram que a variação do albedo é primariamente um resultado da natureza da reflexão da componente direta do espectro de radiação solar. Pelo menos três fatores explicam as diferenças no albedo da superfície. $\mathrm{O}$ primeiro é a própria condição da superfície; em geral, albedos mais altos estão associados com superfícies suaves, secas e de coloração clara, ao passo que albedos mais baixos estão associados com superfícies rugosas, úmidas e coloração escura (Correia et al., 2002). Devido a isso é que o albedo superficial pode ser alterado por ação antropogênica e natural. No caso de áreas cobertas por vegetação, ele depende da altura das plantas, percentagem de cobertura do chão, ângulo das folhas e do índice de área foliar. O segundo fator que controla o albedo é o ângulo zenital do Sol, o que o leva às variações diurnas acentuadas. $\mathrm{O}$ terceiro fator é o estado do céu, com referência particular aos tipos e quantidades de nuvens (Correia et al., 2002; Merikanto et al., 2010; Liu et al., 2011; Leaitch et al., 2010).

O albedo é considerado um dos mais importantes parâmetros na caracterização do clima da terra (Davidson e Wang, 2004; Kirschbaum et al., 2011). Um conhecimento detalhado de como o albedo varia no espaço e no tempo é crucial para entender-se o balanço de radiação global, sua influência no clima e na dinâmica da vegetação (Lucht et al., 2000; Kirschbaum et al., 2011), uma vez que, o aumento ou redução do albedo implica em alteração do balanço radiativo e energético da superfície da terra (Meinander et al., 2008). De acordo com Davidson e Wang (2004), dados de albedo são tão imprescindíveis que os mesmos passam a ser considerados componentes chaves nos modelos de circulação geral da atmosfera. Entretanto, em regiões de altas latitudes, o albedo sofre grande variação no espaço e no tempo, devido a neve que cobre essa região em determinadas épocas do ano, dificultando uma boa performance dos modelos globais (Vikhamar e Solberg, 2003; Kirschbaum et al., 2011).

O impacto no clima em função da alteração no albedo tem sido muito discutido por vários estudiosos da área (Wang, 2005; Kirschbaum et al., 2011; Kushari e Konitpong, 2011; Leaitch et al., 2010). Moura et al. (1999) e Querino et al. (2006), estudaram a variação do albedo em áreas de florestas e pastagem na Amazônia, onde perceberam que os valores médios para regiões de florestas são de aproximadamente $13 \%$, enquanto na pastagem $18 \%$, o que significa um aumento médio de $38 \%$ no albedo quando se altera a cobertura natural por pastagem. Querino et al. (2006), também compararam albedo com ângulo zenital na Amazônia, e notaram influência do ângulo na variação do albedo, para sítios de mesma cobertura vegetal e mesmas condições climáticas.

Tendo em vista que manguezais são ecossistemas extremamente importantes, estes ainda são pouco explorados no que diz respeito ao entendimento da troca de energia e as variáveis que o controlam. Então, o principal objetivo desse trabalho foi avaliar o albedo sobre manguezal por meio de comparação entre duas áreas distintas de mangue, nativa $\mathrm{e}$ degradada, visando analisar o impacto do desmatamento nessa 
variável. Comparou-se também a variação diurna do albedo do interior do mangue com albedo na copa das árvores com o intuito de entender o quanto de energia fica aprisionada no sistema.

\section{MATERIAL E MÉTODOS}

\section{1 Área de estudo}

A Área de Preservação Ambiental (APA) da Ilha de Santa Rita ocupa uma área de 10.346 ha, e situa-se dentro do Complexo Estuarino Lagunar Mundaú-Manguaba. A APA localiza-se entre as latitudes de $9^{\circ} 37^{\prime} 30^{\prime \prime}$ e $9^{\circ} 47^{\prime} 30^{\prime \prime} \mathrm{S}$ e as longitudes de $35^{\circ} 45^{\prime} 00^{\prime \prime}$ e $35^{\circ} 55^{\prime} 00^{\prime}$ " W. Atualmente é composta de $33,39 \%$ de florestas naturais, $38,77 \%$ de ocupações antrópicas e 25,20\% de superfícies líquidas (Nascimento et al., 2009; Querino et al., 2011).

As observações foram realizadas no período compreendido entre outubro de 2004 e setembro de 2005 em dois sítios experimentais, localizados no município de Marechal Deodoro, distante $15 \mathrm{~km}$ ao sul da cidade de Maceió - AL (Figura 1). Os sítios se distinguem pelo tipo de cobertura vegetal, vegetação nativa (manguezal) e área de manguezal desmatada, substituída por coqueiral e vegetação rasteira (capim).

A área de mangue nativo ( $9^{\circ} 42^{\prime} 18^{\prime \prime}$ S e $35^{\circ} 48^{\prime} 32^{\prime \prime}$

W) ocupa uma área de 41,6 ha pertencente ao condomínio residencial Laguna. Caracteriza-se pela predominância de dois tipos de árvores (Avicennia Germinans L. e Rhizophora Mangle L.), cujo dossel atinge uma altura de até 11,5 m (Calado e Souza, 2002; Querino et al., 2011; Moura e Querino, 2010, 2012). O manguezal degradado localiza-se no Sítio Limoeiro,

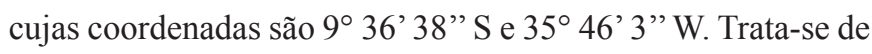
uma propriedade particular com uma área de aproximadamente 31,0 ha onde, praticamente toda ela é destinada a plantação de coqueiro do tipo Híbrido (coqueiro mestiço ou anão gigante). A plataforma para coleta dos dados meteorológicos foi instalada no centro de uma clareira (solo coberto por capim), cercada por esse tipo de coqueiro, com um raio médio de aproximadamente $125 \mathrm{~m}$.

\subsection{Climatologia da região}

O clima da região é considerado tropical, semiúmido com período chuvoso anual compreendido entre os meses de maio e agosto, e época seca entre dezembro e março. A temperatura e a umidade relativa média anual são iguais a $25,5{ }^{\circ} \mathrm{C}$ e $78 \%$, respectivamente. A umidade relativa apresenta seus valores máximos em maio $(82,6 \%)$ e mínimo em novembro $(74,7 \%)$ (Cabús, 2002; Querino et al., 2011; Moura e Querino, 2010, 2012). Contudo, de acordo com Querino et al. (2011), durante o período experimental, os meses considerados mais chuvosos (maio e junho) registraram valores de precipitação superiores ao da normal climatológica. O restante do período experimental apresentou nível de precipitação inferior. A radiação solar média mensal varia de $24 \mathrm{MJ} \mathrm{m}^{-2}$ em julho a $84 \mathrm{MJ} \mathrm{m}^{-2}$ em novembro (Souza et al., 2005).

\subsection{Dados}

Com o intuito de determinar o albedo, medidas de radiação solar incidente $(\mathrm{Rg})$ e refletida $(\mathrm{Rr})$, foram obtidas

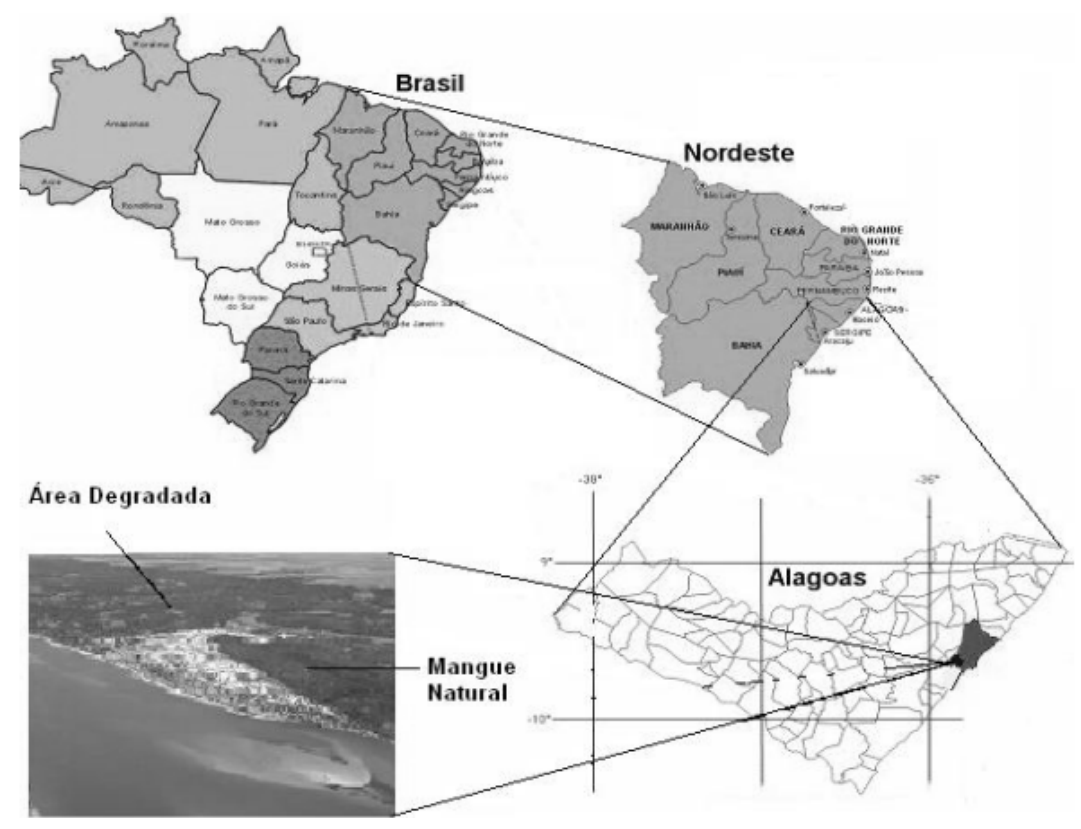

Figura 1 - Mosaico representativo da área de estudo. Em destaque, foto do Complexo estuarino Mundaú-Manguaba. 
em duas plataformas de coletas de dados meteorológicos. $\mathrm{Na}$ área de vegetação nativa, os piranômetros foram posicionados acima da copa, no topo de uma torre de $13 \mathrm{~m}$. Na área degradada, os piranômetros encontravam-se a uma altura de $2 \mathrm{~m}$. Os instrumentos destinados a mensurar a Rg foram do modelo SP - LITE da Campbell Scientific Inc., cujo tempo de resposta é abaixo de 1 segundo, e apresenta um desvio máximo estimado $\mathrm{em} \pm 1 \%$ para cada $1000 \mathrm{~W} \cdot \mathrm{m}^{-2}$ (Querino et al., 2011). Para a medida da radiação refletida foram utilizados dois piranômetros da Campbell Scientific Inc., modelo LI200SZ com suas cúpulas voltadas para baixo. Esses instrumentos apresentam um desvio máximo de $\pm 1 \%$ para cada $3000 \mathrm{~W} \mathrm{~m}^{-2} \mathrm{e}$ são dotados de uma acurácia de $3 \%$. Destaque-se que, no mangue natural em outubro de 2005, foi instalado um instrumento no interior do manguezal, para que se medisse a radiação refletida pela superfície interna do mangue.

\section{RESULTADOS E DISCUSSÃO}

Radiação solar global (Rg) é toda radiação eletromagnética que atinge a superfície da Terra proveniente do Sol. O Rg é responsável direto pelos principais processos de ordem física química e biológica que ocorrem dentro dos mais diversos ecossistemas (Moura et al., 1999; Querino et al., 2006, 2011). Por se tratar de uma variável indispensável também no estudo do albedo, faz-se necessário o entendimento da variação anual do Rg sobre a área de estudo.
A região em estudo tem um ciclo diário médio para $\mathrm{Rg}$ de $12 \mathrm{~h}$ (Figura 2). Máximos médios horários de $900 \mathrm{~W} \mathrm{~m}^{-2}$ durante os meses compreendidos entre setembro e março, no intervalo das 10 às $14 \mathrm{~h}$, foram registrados na área de estudo. Para os meses entre abril e julho o valor médio máximo atingido foi de $\sim 600 \mathrm{~W} \mathrm{~m}^{-2}$. A razão para essa diferença entre os valores médios máximos pode ser atribuída a fatores astronômicos, como declinação solar e ângulo zenital. O caminho ótico a ser percorrido pelos raios solares provoca redução na quantidade de radiação que chega a superfície, quanto maior o ângulo zenital, maior atenuação pela atmosfera (Querino et al., 2006, 2011). Como os horários compreendidos entre 10 e $14 \mathrm{~h}$ apresentam menor ângulo zenital, é compreensível que os máximos sejam atingidos dentro desse intervalo.

Observou-se também que o $\mathrm{Rg}$ médio horário não foi simétrico durante todo o ano. De acordo com Querino et al. (2011) essas flutuações diárias são atribuídas às diferentes condições de turbidez da atmosfera. Ainda, segundo esses autores, durante o período matutino a transmitância atmosférica para a região em questão é maior do que $70 \%$, enquanto que no período vespertino a transmitância não atinge $50 \%$.

A radiação que atinge a superfície, seja ela incidente, refletida ou absorvida, é de extrema importância para se avaliar possíveis impactos microclimáticos que a modificação da cobertura de qualquer floresta natural, pode causar. Por isso, o estudo comparativo entre o albedo em áreas de florestas nativas e degradadas é crucial na avaliação de possíveis

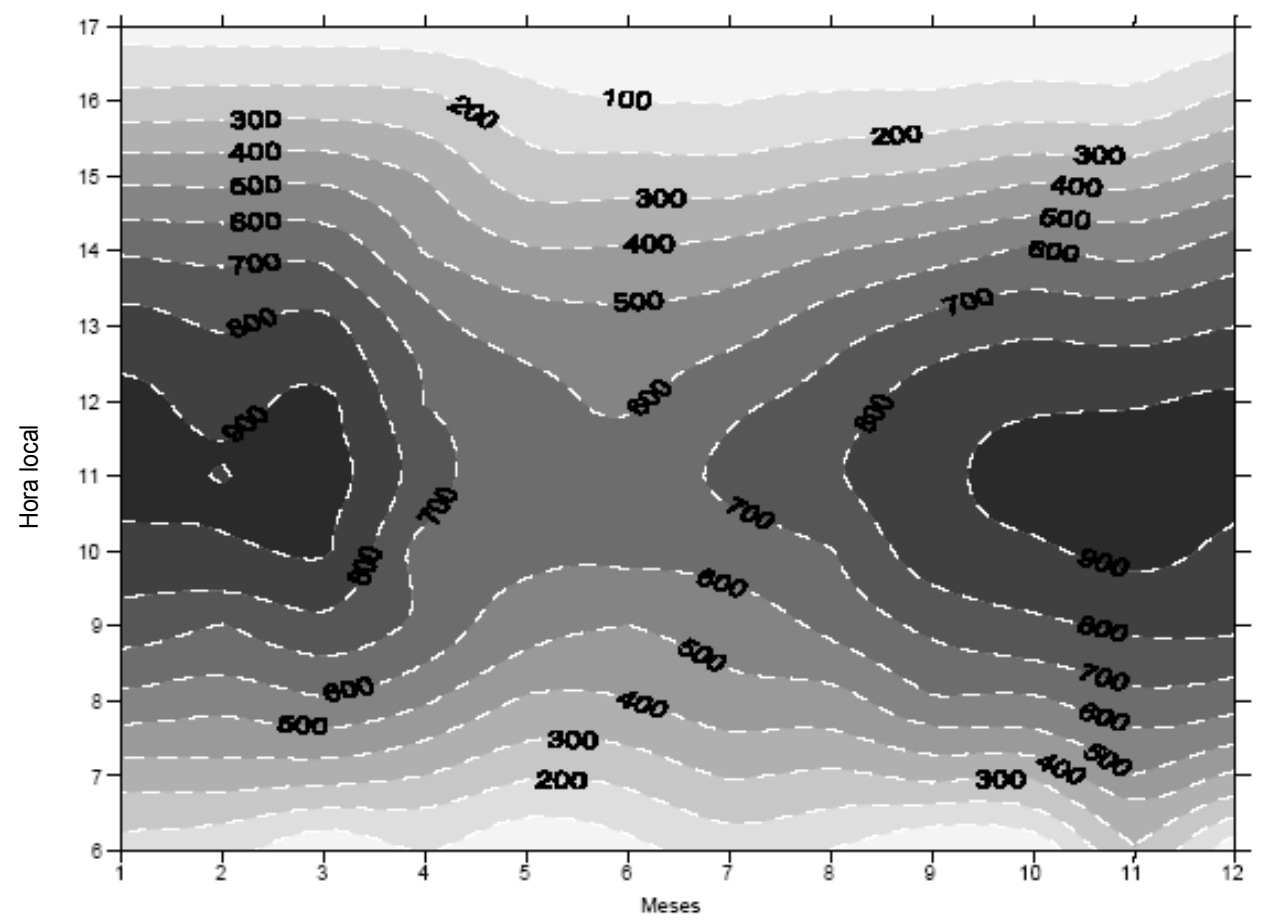

Figura 2 - Radiação solar global (Rg) (W.m-2). Ciclo de médias horárias mensal compreendido entre os meses de outubro de 2004 e setembro de 2005. 
alterações no balanço radiativo e consequentemente mudanças no microclima.

Verificou-se que durante praticamente todo ano no horário entre 9 e 14h o Alb_Nat foi, em média, de 18\% (Figura 3). O fato de permanecer constante ocorre porque de acordo com Moura et al. (1999), a assimetria da curva do albedo para áreas de floresta não tem relação direta com o ângulo zenital e estaria mais relacionada à geometria da copa ou ao tipo de planta.

Essa relação com o tipo de planta e a geometria da copa, pode ser o motivo pelo qual o albedo, para a floresta de mangue, em média, é maior do que em florestas tropicais, como por exemplo, a Amazônia. Por apresentar um dossel mais denso a Amazônia tende a absorver mais radiação do que refletir, quando comparado com o mangue em questão. Querino et al. (2006) e Moura et al. (1999) verificaram que o albedo sobre o dossel na Amazônia é em média de $13 \%$, ou seja, aproximadamente 5 pontos percentuais mais baixo do que os valores médios para esse estudo, e 10 pontos percentuais mais baixo do que a média máxima encontrada que foi de $24 \%$.

Ainda na área de mangue nativo, realizou-se uma comparação entre o albedo sobre a copa do mangue e o albedo na superfície interna durante o mês de outubro de 2005 (Figura 4). Nota-se que a fração interna é bem menor do que a externa. O valor médio máximo não ultrapassa $13 \%$. A razão para isso está na coloração escura do solo resultante da decomposição de matéria orgânica (Querino, 2006; Moura e Querino, 2011,
2012). Salienta-se, ainda, que esse aumento ocorreu no horário de menor média do albedo externo.

O fato pode está relacionado a dois motivos: o primeiro seria com relação a uma maior penetração da radiação solar nesse horário, que de acordo com Querino et al. (2011) os elevados valores de incidência da radiação na superfície interna do mangue é decorrente da passagem do Sol no zênite local, diminuindo dessa forma o ângulo de incidência, o que facilitaria a penetração da radiação por entre o dossel do mangue. $\mathrm{O}$ segundo motivo estaria relacionado com a altura da maré. Moura e Querino (2012) detectaram 21 dias, no mês de outubro, onde a maré nos horários entre as 10 e às 14 horas estaria cheia, enchendo, ou nem muito seca nem muito cheia (popular maré morta). Nessas condições, lâminas d'água na superfície do manguezal foram formadas. Com isso, a intensificação do reflexo da radiação que atingiu a superfície do manguezal, ocasionou o aumento do albedo interno nesse horário.

$\mathrm{Na}$ área degradada o albedo apresenta grande variação entre as distintas épocas do ano. Os meses considerados secos, entre dezembro e março, são os que apresentam maiores médias. A elevação dos valores está atribuída a um solo, e, por consequência, a uma vegetação mais ressecada. Dessa forma, além do alto poder de reflexão do próprio solo degradado, tem-se para essa época, uma maior concentração de vegetação com folhagens mais claras ou acinzentadas, o que eleva a reflexão (Figura 5). Outro fator que contribui para o aumento do albedo

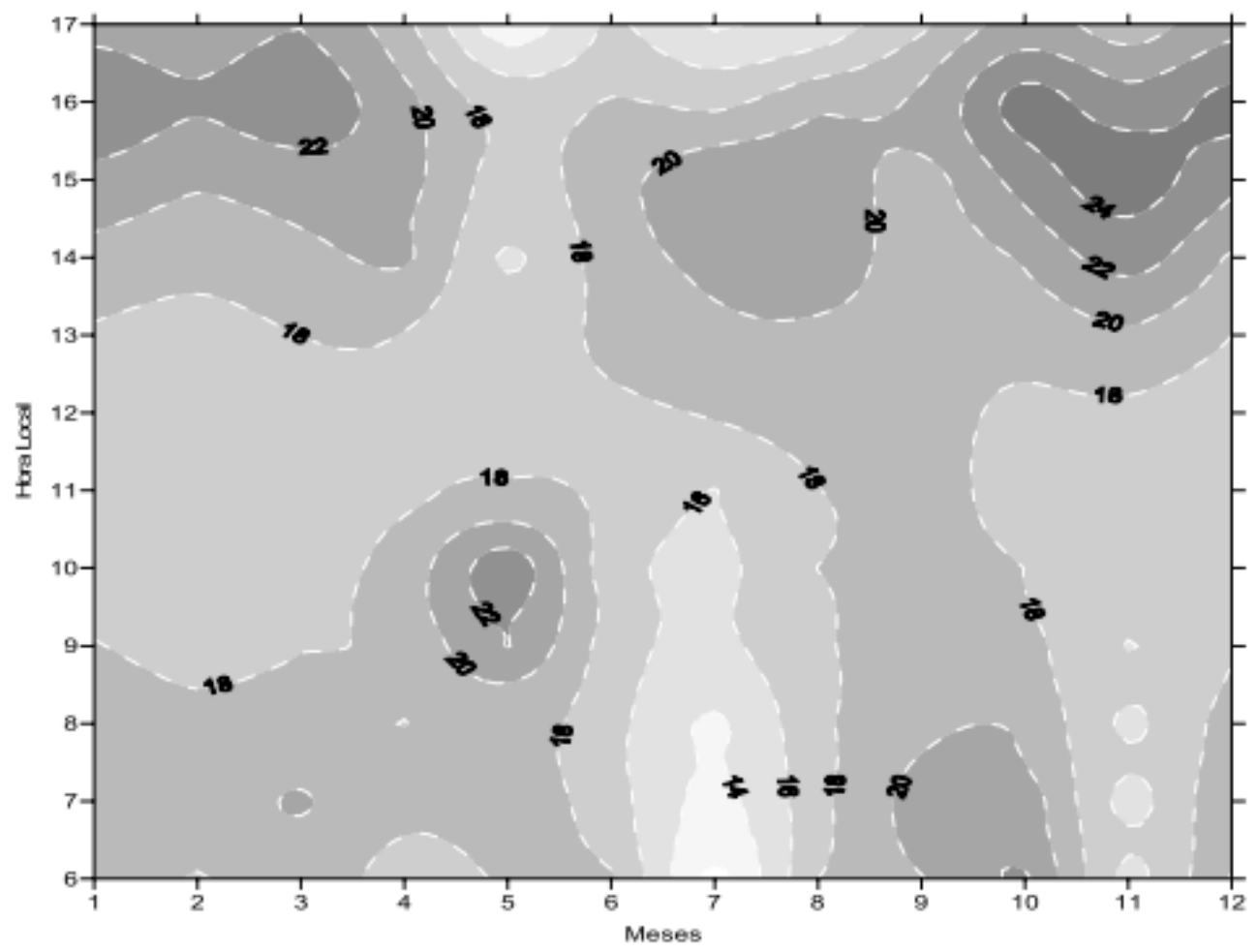

Figura 3 - Albedo médio mensal (\%) sobre a área de mangue nativo para o período de estudo. 


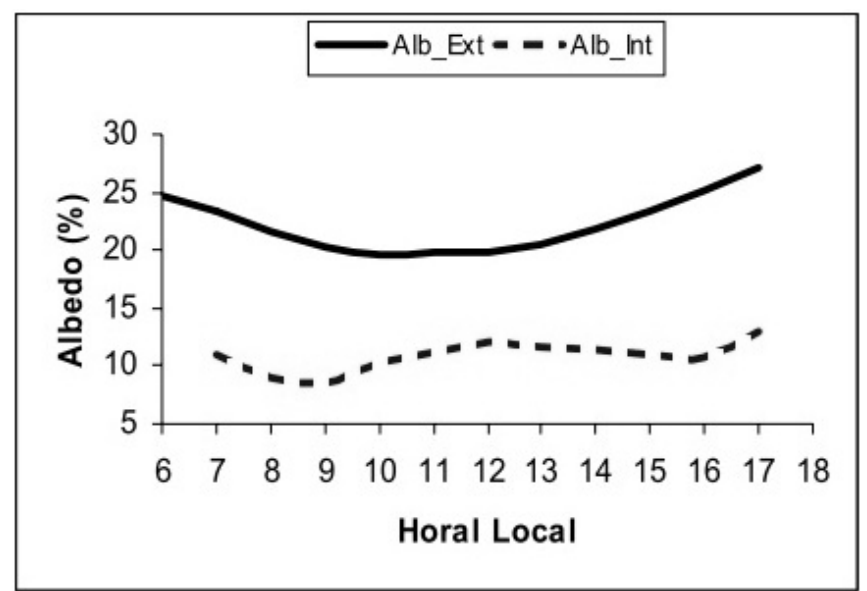

Figura 4 - Albedo médio (\%) no interior do mangue natural e acima da copa das árvores durante o mês de outubro de 2005.

foi diagnosticado por Moura e Querino (2010; 2012). Os autores detectaram que o solo nessa área é composto em grande parte de areia branca (86\%), aumentando dessa forma o poder reflexivo da superfície em questão.

A estação chuvosa apresentou valores de albedo inferiores aos da estação seca. Durante toda a quadra chuvosa o valor médio de albedo foi em torno de $30 \%$. Os períodos de transição, seco-chuvoso e chuvoso-seco, registraram valores distintos. Para março (seco-chuvoso), o albedo atingiu $40 \%$. Esse valor é superior aos dos meses setembro e outubro (20 e 30\%), meses que obtiveram os menores registros de albedo para área degradada. Uma explicação plausível pode ser dada por conta da vegetação rasteira seca, que ainda dominava a área da estação meteorológica no mês de março. Já em setembro, verificou-se que ocorreu uma floração e um crescimento da vegetação, próxima da torre de coleta, deixando a superfície refletora mais escura e que perdurou até o mês de outubro.

Quando se substitui floresta nativa por uma cobertura de grama (pastagem), em média, o albedo eleva-se de 13\% para $18 \%$, como foi detectado por Moura et al. (1999) e Querino et al. (2006), em estudos realizados na Amazônia. Salienta-se, porém, que a área de mangue degradado, com o ressecamento do solo, tende a adquirir características mais claras dando lugar a uma cobertura que mistura resto de mangue com areia de praia, o que eleva o albedo (média de $35 \%$ ).

Ao observar-se a diferença entre o albedo médio na área nativa, (18\%) e na área degradada (35\%), percebe-se uma elevação significativa do albedo quando se altera a cobertura natural. Isso significa um aumento de $49 \%$ na quantidade de energia refletida para a atmosfera e que deveria ficar na superfície para os mais diversos processos físico, químico e biológico.

\section{CONCLUSÕES}

Diante dos resultados obtidos e expostos anteriormente e as discussões atribuídas a eles, enunciam-se as seguintes conclusões:

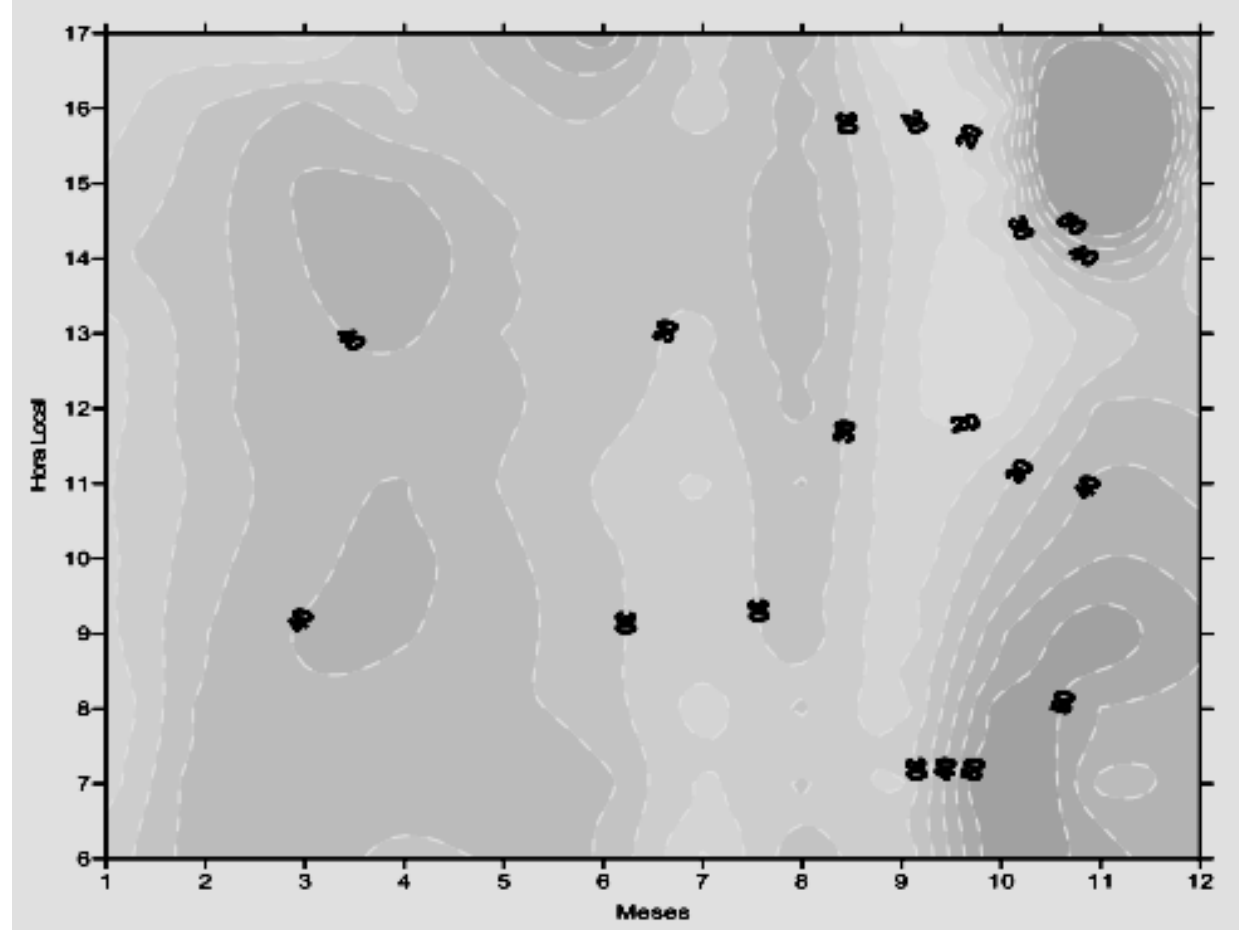

Figura 5 -Albedo médio mensal (\%) sobre a área de mangue degradado para o período de estudo. 
- O albedo sobre floresta de mangue, em geral, é maior do que em outras florestas tropicais, média de $18 \%, 5$ pontos percentuais superior em relação, por exemplo, ao da floresta tropical amazônica (13\%).

- Internamente o albedo não ultrapassou os $13 \%$ e seu valor máximo ocorre no horário de menor albedo da copa, evidenciando a influência da maré.

- $\quad \mathrm{Na}$ área degradada, o albedo médio foi de 35\%, o que implica em uma elevação aproximada de $49 \%$, quando se substitui a cobertura de floresta natural.

\section{REFERÊNCIAS}

CABÚS, R.C. Tropical daylighting: predicting sky types and interior illuminances in North - East Brazil. Reino Unido, 2002. Cáp.3, 14p. Tese (PhD em Arquitetura) - Universidade de Sheffield - School of Architectural Studies.

CALADO, T.C.S; SOUSA, C.E. Crustáceos do complexo estuarino - lagunar Mundaú/Manguaba Alagoas. Maceió: FAPEAL, 116p. 2002.

CORREIA, F.W.S.; ALVALÁ, R.C. DOS S.; GIELOW, R.; MANZI, A.O.; SOUZA, A. de. Albedo do Pantanal Sul Matogrossense durante o período de transição seco - úmido de 2001 - 2002. In: Congresso Brasileiro de Meteorologia, 12, 2002 Foz do Iguaçu - PR. Anais... 1 CD ROM.

DAVIDSON, A.; WANG, S. The effects of sampling resolution on the surface albedos of dominant land cover types in the North American boreal region. Remote Sensing of Environment, v. 93, p. 211- 224. 2004.

GALVANI, E.; DE LIMA, N.G.B. Radiação solar acima e abaixo de dossel de manguezal na Barra Do Ribeira do Iguape, SP. Revista Geográfica Acadêmica, v.5, n.1, p. 59 - 67, 2011. HOGARTH, P. The biology of mangroves and seagrasses. New York, Oxford University press, 273p, 2007.

KIRSCHBAUM, M. U. F.; WHITEHEAD, D.; DEAN, S. M.; BEETS, P. N.; SHEPHERD, J. D.; AUSSEIL, A.-G. E. Implications of albedo changes following afforestation on the benefits of forests as carbon sinks. Biogeosciences, v.8, p.3687-3696, 2011.

KUSHARI, B.; KANITPONG, K. Surface Albedo of Bangkok Roads. Proceedings of the Eastern Asia Society for Transportation Studies, Vol.8, 2011.

LEAITCH, W. R.; LOHMANN, U.; RUSSELL, L. M.; GARRETT, T.; SHANTZ, N. C.; TOOM-SAUNTRY, D.; STRAPP, J. W.; HAYDEN, K. L.; MARSHALL, J.; WOLDE, M.; WORSNOP, D. R.; JAYNE, J. T. Cloud albedo increase from carbonaceous aerosol. Atmospheric Chemistry and Physics, v.10, p.7669-7684, 2010.

LIU, Y.; WU, W.; JENSEN, M. P.; TOTO, T.. Relationship between cloud radiative forcing, cloud fraction and cloud albedo, and new surface-based approach for determining cloud albedo. Atmospheric Chemistry and Physics, v.11, p. 7155-7170, 2011.

LUCHT, W.; SCHAAF, C.B.; STRAHLER, A. H. An algorithm for the retrieval of

albedo from space using semiempirical BRDF models IEEE.

Rev. Transactions on Geoscience and Remote Sensing. v. 38, n.2. p. 977-998. 2000.

MEINANDER, O; KONTU, A.; LAKKALA, K.; HEIKKILÄ, A.; YLIANTTILA, L.; TOIKKA, M. Diurnal variations in the UV albedo of arctic snow. Atmospheric Chemistry and Physics, v.8, p. 6551-6563, 2008.

MENDOZA-CARRANZA, M.; HOEINGHAUS, D.J; GARCIA, A.M; ROMERO-RODRIGUEZ, A. Aquatic food webs in mangrove and seagrass habitats of Centla Wetland, a Biosphere Reserve in Southeastern Mexico. Neotropical Ichthyology, v.8, n.1 p. 171-178, 2010.

MERIKANTO, J.; SPRACKLEN, D. V.; PRINGLE, K. J.; CARSLAW, K. S. Effects of boundary layer particle formation on cloud droplet number and changes in cloud albedo from 1850 to 2000. Atmospheric Chemistry and Physics, v.10, p.695-705, 2010.

MOURA, M.A.L. Balanço de Radiação à superfície e fluxo de calor no solo em áreas de floresta nativa e pastagem no Oeste da Amazônia brasileira. Botucatu, 2000. 118p. Tese (Doutorado em Agronomia). Faculdade de Ciências Agronômicas da UNESP.

MOURA, M.A.L.; LYRA, R.F.F.; BENINCASA, M.; SOUZA, J.L.; NASCIMENTO FILHO, M.F. Variação do albedo em áreas de floresta e pastagem na Amazônia. Revista Brasileira de Agrometeorologia, v.7, n.2, p.163 - 168, 1999.

MOURA, M.A.L; QUERINO, C.A.S. Evaluation of the seasonal variation of the soil temperature as function of the influence of the tide and precipitation in a tropical mangrove. International Journal of Ecology \& Development, v.21, n.1, p.1-14, 2012.

MOURA, M.A.L; QUERINO, C.A.S. Variação sazonal do fluxo de calor no solo dentro de um manguezal tropical. Revista Brasileira de Engenharia Agrícola e Ambiental, v.14, n.3, p.296-302, 2010.

NASCIMENTO, M.C.; SILVA, M.P.; GUIMARAES JUNIOR, S.A.M. Geoprocessamento aplicado a análise dos impactos ambientais na cobertura vegetal da Área de Proteção Ambiental de Santa Rita, Alagoas-Brasil.In: Anais XIV Simpósio Brasileiro de Sensoriamento Remoto, XIV, 2009, Natal - RN, Anais.... p. 4101-4108.

OSÓRIO, F.M; GODINHO, W.O; LOTUFO, T.M.C. Ictiofauna associada às raízes de mangue do estuário do Rio Pacoti CE, Brasil. Biota Neotropica, v.11, n.1, p.415-420, 2011. 
PINKER, R.T.; THOMPSON, E.; ECK, T.F. The albedo of a tropical evergreen forest. Quart. Journal of Meteorological Society, v.106, p.551-558, 1980.

QUERINO, C.A.S.; MOURA, M.A.L., LYRA, R.F.F. MARIANO, G.L. Avaliação e comparação de radiação solar global e albedo com ângulo zenital na região Amazônica. Revista Brasileira de Meteorologia, v.21, n.3a, p.42-49, 2006.

QUERINO, C.A.S.; MOURA, M.A.L.; QUERINO, J.K.A.S.; VON RADOW, C.; MARQUES FILHO, A.O. Estudo da radiação solar global e do índice de transmissividade (kt), externo e interno, em uma floresta de mangue em Alagoas - Brasil. Revista Brasileira de Meteorologia, v.26, n.2, p.204-294, 2011.

RIBEIRO, J.B.M.; DOS SANTOS, C.V.; CHAVES, J.G.; MATTOS, A.; RODRIGUES, H.J.B. Característica do vento acima da copa das árvores no manguezal de Bragança - PA. In: Congresso Brasileiro de Meteorologia, 12, 2002 Foz do Iguaçu - PR. Anais... 1 CD ROM.

SATHIA, T.; SEKAR, C. Mangrove Eco-system and their multifunctionalities: an analysis of the provisions of economic and environmental livelihoods to the fisherman communities in the south-east coast of India. Trends in Agricultural Economics, v.5, n. 2, p. 31 - 47, 2012.
SOUZA, J.L. DE.; NICÁCIO, R.M.; MOURA, M.A.L. Global solar radiation measurements in Maceió, Brazil. Renewable Energy, v.30, p.1203-1220, 2005.

STANHILL, G.; HOFSTEDE, G.J.; KALMA, J.D. Radiation balance of natural and agricultural vegetation. Quart. Journal of Meteorological Society, v.92, p.128-40, 1966. SWEETMAN A.K.; MIDDELBURG, J.J.; BERLE, A.M; BERNARDINO, A.F.; SCHANDER, C.; DEMOPOULOS, A.W.J.; SMITH, C.R. Impacts of exotic mangrove forests and mangrove deforestation on carbon remineralization and ecosystem functioning in marine sediments. Biogeosciences, v.7, p. 2129-2145, 2010.

VIKHAMAR, D.; SOLBERG, R. Subpixel mapping of snow cover in forests by optical

remote sensing. Rev. Remote Sensing of Environment. v. 84, p. 69-82, 2003

WANG, S. Dynamics of surface albedo of a boreal forest and its simulation. Ecological Modelling, v.183, p.477-494. 2005. 